\title{
METADATA AUTHORITY DESCRIPTION SCHEMA (MADS): UMA ALTERNATIVA À UTILIZAÇÃO DO FORMATO MARC 21 PARA DADOS DE AUTORIDADE*
}

\section{METADATA AUTHORITY DESCRIPTION SCHEMA (MADS): UNA ALTERNATIVA AL USO DEL FORMATO MARC 21 PARA DATOS DE AUTORIDAD}

\begin{abstract}
Fabrício Silva Assumpção - assumpcao.f@gmail.com Mestrando em Ciência da Informação pela Universidade Estadual Paulista (UNESP/Marília). Bacharel em Biblioteconomia.

Plácida Leopoldina Ventura Amorim da Costa Santos placida@marilia.unesp.br Livre-docente em Catalogação pela Universidade Estadual Paulista (UNESP/Marília). Docente do Departamento de Ciência da Informação da Universidade Estadual Paulista (UNESP/Marília)
\end{abstract}

\section{RESUMO}

Introdução: O intercâmbio de registros de autoridade requer o estabelecimento e a adoção de padrões de metadados, tais como o Formato MARC 21 para Dados de Autoridade, formato utilizado por diversas agências catalogadoras, e o Metadata Authority Description Schema (MADS), padrão que permanece pouco explorado pela literatura e pouco difundido entre as agências.

Objetivo: Apresentar um estudo introdutório sobre o padrão MADS.

Metodologia: Pesquisa bibliográfica descritiva e exploratória.

Resultados: São abordados o contexto de criação do MADS, seus objetivos, sua estrutura e as principais questões relacionadas à conversão de registros em MARC 21 para registros MADS.

\footnotetext{
* Estudo desenvolvido com financiamento da Fundação de Amparo à Pesquisa do Estado de São Paulo (FAPESP).
} 
Conclusões: Conclui-se que, apesar de suas limitações, o MADS pode ser utilizado para a criação de registros de autoridade simples, no ambiente Web e além do contexto das bibliotecas.

Palavras-chave: Metadata Authority Description Schema (MADS). Extensible Markup Language (XML). Formato MARC 21 para dados de autoridade. Controle de autoridade. Catalogação descritiva. Informação e tecnologia.

\section{INTRODUÇÃO}

No século XIX, Charles Coffin Jewett defendeu que um livro deveria ser catalogado uma única vez, não sendo necessário que uma biblioteca catalogasse um recurso que já havia sido catalogado por outra biblioteca. Tal anseio - um recurso informacional ser catalogado uma única vez - tornou-se evidente na catalogação, dando origem aos sistemas de alimentação de catálogos por meio dos programas de catalogação centralizada e de catalogação cooperativa.

Entretanto, para a concretização do que foi defendido por Jewett e a efetivação dos sistemas de alimentação de catálogos em um processo de intercâmbio de dados bibliográficos e de interoperabilidade entre os sistemas, o estabelecimento e a adoção de padrões de representação são uma exigência. Padrões esses, representados pelos padrões de metadados, podem ser agrupados em várias categorias de acordo com as funções que desempenham.

Na categorização de Alves (2010, p. 92) existem os padrões de estrutura de dados, os quais apresentam um conjunto de elementos prescritos, a definição desses elementos e um comentário do que deve ser apresentado.

Dentre os padrões de estrutura de dados utilizados para o intercâmbio de registros bibliográficos estão o MARC 21 Format for Bibliographic Data (Formato MARC 21 para Dados Bibliográficos) e o Metadata Object Description Schema (MODS) (Esquema de Metadados para a Descrição de Objeto).

Um dos motivos que levaram a busca por alternativas para que um recurso informacional fosse catalogado uma única vez foi o custo da catalogação. O compartilhamento da catalogação reduziria o tempo gasto pelo catalogador, o que resultaria, entre outros, em uma redução dos gastos com a catalogação.

Além de ocupar-se com os registros bibliográficos, a catalogação ocupa-se também com os registros de autoridade, os quais são utilizados principalmente para 
registrar os pontos de acesso (autorizado e remissivas) de uma entidade, apoiando à utilização do catálogo e permitindo que seja alcançado o controle de autoridade.

A criação de registros de autoridade mostra-se como algo de alto custo, pois compreende atividades que podem demandar um grande período de tempo em sua realização, tais como a atividade de consulta à fontes de informação para descobrir quais nomes são utilizados pela entidade e a construção de seu ponto de acesso autorizado. Dessa forma, quando criados, os registros de autoridade devem ser mantidos pela agência catalogadora, possibilitando seu uso e reuso.

Uma vez que registros de autoridade são criados e mantidos, é possível intercambiar tais registros entre as agências, o que evitaria que mais de um registro de autoridade, representando uma mesma entidade e de acordo com as mesmas regras de catalogação, precisasse ser elaborado.

Para possibilitar o intercâmbio de registros de autoridade foram criados vários padrões. Um desses padrões é o MARC 21 Format for Authority Data (Formato MARC 21 para Dados de Autoridade), já conhecido e utilizado por diversas agências, inclusive no Brasil. No entanto, há um padrão para o intercâmbio de registros de autoridade que permanece pouco explorado pela literatura, principalmente pela literatura nacional, e pouco difundido entre as agências catalogadoras. Esse padrão, que tem em sua base a Extensible Markup Language (XML) (Linguagem de Marcação Extensível), é o Metadata Authority Description Schema (MADS) (Esquema de Metadados para a Descrição de Autoridade).

Diante da falta de estudos, principalmente em âmbito nacional, sobre o MADS, tem-se como objetivo, por meio de uma pesquisa bibliográfica descritiva e exploratória, apresentar um estudo introdutório sobre o MADS, abordando o contexto de sua criação, seus objetivos, sua estrutura e as principais questões relacionadas à conversão de registros MARC 21 para registros MADS.

\section{CONTROLE DE AUTORIDADE}

Uma entidade, entendida aqui como "algo com caráter unitário e independente; algo com existência independente ou separada; uma abstração, conceito ideal, objeto de pensamento ou objeto transcendental" (INTERNATIONAL FEDERATION OF LIBRARY ASSOCIATIONS, 2009, p. 11, tradução nossa), pode 
ser conhecida por mais de um nome ou por diferentes formas de seu nome, assim como um nome pode referir-se a mais de uma entidade. Esses distintos nomes e suas diversas formas são utilizados como base para a elaboração de pontos de acesso e são utilizados para encontrar recursos informacionais representados nos catálogo.

Diante da diversidade de pontos de acesso para uma entidade, um usuário do catálogo com o conhecimento de apenas um dos nomes pode não encontrar todos os recursos informacionais associados à entidade por ele desejada, por exemplo, todos os livros de um determinado autor. Desse modo, faz-se necessário reunir e controlar os pontos de acesso, assegurando que tal usuário encontre todos os recursos associados à entidade desejada ao realizar uma única busca. Dessa necessidade surge o conceito de controle de autoridade.

Taylor (1984, p. 2 apud MARAIS, 2004, p. 62, tradução nossa) define controle de autoridade como "o processo de manter a consistência nos cabeçalhos [pontos de acesso] em um arquivo bibliográfico por meio de referências a um arquivo de autoridade", enquanto que Burger (1985) o define como o estado alcançado quando os pontos de acesso em um catálogo estão consistentes e quando um mecanismo (o arquivo de autoridade) e políticas de catalogação têm sido estabelecidos para assegurar essa consistência.

Um arquivo de autoridade pode ser definido como uma coleção de registros de autoridade. Reitz (2010, tradução nossa) complementa:

Uma lista de formas autorizadas de cabeçalhos utilizados em um catálogo de biblioteca ou arquivo de registros bibliográficos, mantido para assegurar que os cabeçalhos são aplicados consistentemente quando novos itens são adicionados ao acervo. Usualmente, são mantidos arquivos de autoridade separados para nomes, títulos uniformes, títulos de séries e assuntos. Todas as remissivas feitas para e de um dado cabeçalho também são incluídas no arquivo.

Marais (2004, p. 68) aponta algumas vantagens decorrentes do controle de autoridade e da criação de arquivos de autoridade:

- Arquivos de autoridade proporcionam um melhor acesso à informação: a utilização de arquivos de autoridade conduz a uma maior precisão e exatidão em sistemas de informação e permite recuperar todos os itens 
sobre uma determinada entidade. Um melhor acesso provê um serviço melhor ao usuário: sem o controle de autoridade o usuário precisa considerar todos os possíveis pontos de acesso para uma entidade.

- Arquivos de autoridade vinculam pontos de acesso: a utilização de um arquivo de autoridade é um dos meios para vincular ou reunir pontos de acesso relacionados. As formas variantes (remissivas) conduzem os usuários aos pontos de acesso autorizados e também proveem uma estrutura lógica que facilita o uso do catálogo.

- Arquivos de autoridade promovem o controle bibliográfico e são requeridos para tal.

- Arquivos de autoridade contribuem para uma boa qualidade do catálogo: os arquivos de autoridade requerem que os catalogadores sigam regras e procedimentos, resultando em menos erros, na eliminação de registros duplicados e de pontos de acesso desnecessários.

A base para a criação de um arquivo de autoridade e, consequentemente, para o controle de autoridade, são os registros de autoridade. A Declaração dos Princípios Internacionais de Catalogação define um registro de autoridade como "um conjunto de elementos de dados que identifica uma entidade e pode ser utilizado para facilitar o acesso ao ponto de acesso autorizado para tal entidade ou a exibição de qualquer ponto de acesso para a entidade." (INTERNATIONAL FEDERATION OF LIBRARY ASSOCIATIONS, 2009, p. 9, tradução nossa).

Uma vez que as agências catalogadoras criam e mantêm registros de autoridade, é possível intercambiar esses registros, como grande parte das agências faz com seus registros bibliográficos. Para tal intercâmbio, no entanto, faz-se necessário estabelecer e adotar certos padrões, dentre os quais estão os padrões de estrutura de dados anteriormente mencionados.

\section{OS FORMATOS MARC 21 E O MARC 21 XML SCHEMA}

Atualmente, um dos padrões de estrutura de dados utilizados para o intercâmbio de registros na comunidade de bibliotecas é o Formato MARC 21 para Dados Bibliográficos. O MARC 21 bibliográfico, assim como o Formato MARC 21 
para Dados de Autoridade, é um dos muitos formatos derivados do formato Machine-Readable Cataloging (MARC) (Catalogação Legível por Máquina), desenvolvido na década de 1960 por Henriette Avram na Library of Congress (LC) (TAYLOR; JOUDREY, 2009).

A estrutura projetada especialmente para a comunicação entre sistemas de processamento de dados de um registro MARC 21, seja ele um registro bibliográfico, de autoridade ou de qualquer um dos outros formatos MARC 21, é baseada na norma ISO 2709 Documentation - Format for Bibliographic Information Interchange on Magnetic Tape (Documentação - Formato para intercâmbio de informação bibliográfica em fita magnética) que torna os registros gerados portáveis entre sistemas.

Durante o intercâmbio de um registro MARC 21, a cadeia de caracteres que o compõe é exportada de um software (por exemplo, um sistema de gerenciamento de bibliotecas) e importada por outro, podendo esse processo ser realizado por meio de diversas interfaces. O registro, ao chegar ao software de destino, é então processado.

Embora os formatos MARC 21 tenham pouco mais de dez anos de existência, eles descendem do MARC desenvolvido na década de 1960. Tal fato faz com que os formatos, embora tenham passado, e ainda estejam passando, por inúmeras atualizações, tenham sua estrutura pautada em uma tecnologia que muitas vezes não condiz com tecnologias mais avançadas na utilização do potencial por elas oferecido, o que tem incitado diversas discussões e críticas no cenário, principalmente internacional, da catalogação, tais como as de Miller e Clarke (2004, p. 102-116).

Em 1986 a International Organization for Standardization (ISO) (Organização Internacional para Padronização) reconheceu como um padrão a Standard Generalized Markup Language (SGML) (Linguagem Padronizada de Marcação Genérica), linguagem criada para facilitar a produção e o compartilhamento de documentos eletrônicos (MILLER; CLARKE, 2004, p. 2). Tendo como base a SGML, em 1998, a Extensible Markup Language (XML) (Linguagem de Marcação Extensível) foi lançada pelo World Wide Web Consortium (W3C).

Originalmente projetada para vencer os desafios da publicação eletrônica em larga escala, a XML hoje está adquirindo um papel cada vez mais importante no 
intercâmbio de uma variedade de dados na Web e em outros ambientes digitais (WC3, 2011).

Segundo Ray (2003, p. 6, tradução nossa),

\begin{abstract}
Estritamente falando, a XML não é uma linguagem de marcação. Uma linguagem tem vocabulário e gramática fixados, a XML na verdade não define qualquer elemento. Em vez disso ela estabelece uma série de restrições sintáticas sob as quais você pode construir sua própria linguagem. Assim, uma descrição mais adequada seria chamar a XML de um kit de ferramentas para linguagem de marcação.
\end{abstract}

Para Siqueira (2003, p. 71), a XML "é uma linguagem para criar padrões de comunicação entre sistemas de computadores, o que permitirá a integração tanto da base de dados como de arquiteturas, hardwares e métodos de programação usados, favorecendo a interoperabilidade".

Visando a possibilitar a utilização da SGML no intercâmbio de registros MARC, a LC desenvolveu na década de 1990 duas Document Type Definitions (DTD) para a conversão de registros nos formatos USMARC (versão do formato MARC vigente na época) para a SGML e da SGML para os formatos USMARC (LIBRARY OF CONGRESS, 2011c).

Uma DTD é utilizada para definir a estrutura de um tipo de documento; ela apresenta, dentre outros, uma lista dos elementos que são válidos para um documento de determinado tipo. Assim, as DTDs SGML do USMARC definiam quais campos e subcampos poderiam ocorrer em registros USMARC.

A primeira DTD criada pela LC destinava-se aos registros bibliográficos, de coleção e de informação para a comunidade, enquanto que a segunda cobria os registros de autoridade e de classificação.

O lançamento da XML em 1998, o surgimento do MARC 21 e os demais avanços tecnológicos tornaram necessária a atualização das DTDs existentes, o que resultou na criação de DTDs XML para o MARC 21 (FLAMINO, 2006, p. 122).

As DTDs XML dos formatos MARC 21 definiam todos os elementos que poderiam aparecer em um registro MARC 21 em XML e especificavam como esses elementos seriam rotulados e representados com a codificação da XML (TAYLOR; JOUDREY, 2009, p. 153). 
Em 2002, com o objetivo de dar continuidade aos esforços empreendidos para a utilização de registros nos formatos MARC 21 em ambientes que utilizam a XML, a LC lançou o MARC 21 XML Schema, mais conhecido como MARCXML.

Um esquema XML, ou seja, um documento construído de acordo com a $X M L$ Schema, desempenha um papel semelhante ao de uma DTD: define a estrutura do documento, os elementos permitidos, etc. Para Taylor e Joudrey (2009, p. 153), a $X M L$ Schema surgiu como uma forma mais rica das DTDs, definindo o conteúdo e as semânticas do documento, em adicional à definição de sua estrutura.

Junto ao MARCXML, que permite a marcação completa em XML de registros nos formatos MARC 21, são disponibilizadas pela LC ferramentas e folhas de estilo que permitem converter os registros MARC 21 que estão estruturados de acordo com a ISO 2709 para registros MARC 21 em XML e estes em registros de acordo com outros padrões de metadados (TAYLOR; JOUDREY, 2009, p. 153).

Assim como existem críticas aos tradicionais formatos MARC 21 (MORENO; BRASCHER, 2007, p. 18), existem críticas ao MARCXML. Miller e Clarke (2004, p. 103, tradução nossa) apontam que:

Os recentes esforços para modernizar o MARC tem, em sua maioria, envolvido uma tradução literal e sem crítica dos campos, indicadores e subcampos para elementos e atributos XML. Infelizmente, isso incorpora no novo meio os problemas inerentes ao MARC e não permite que se faça uso das reais vantagens da XML.

Além de adaptar os formatos MARC 21 visando ao seu uso em ambientes que utilizam a XML, por exemplo, a Web, a LC, ao longo da última década, desenvolveu padrões que em sua origem têm como base a XML e podem apresentar uma alternativa à utilização dos formatos MARC 21.

\section{O METADATA OBJECT DESCRIPTION SCHEMA (MODS) E O SURGIMENTO DO MADS}

Em 2002, foi criado pela LC o Metadata Object Description Schema (MODS) (Esquema de Metadados para a Descrição de Objeto), um esquema XML com um conjunto de elementos bibliográficos que pode ser utilizado para uma variedade de propósitos (LIBRARY OF CONGRESS, 2010b). 
O MODS inclui um subconjunto de elementos do MARC 21 bibliográfico permitindo a representação em XML dos dados de um registro bibliográfico em MARC 21, tem como propósito a conversão dos dados essenciais do registro bibliográfico, podendo alguns dados específicos serem descartados durante a passagem de um padrão para outro. Enquanto um conjunto de elementos para a descrição original de recursos, o MODS permite que um registro simples seja criado utilizando elementos mais genéricos que os disponíveis no MARC 21 bibliográfico (LIBRARY OF CONGRESS, 2010b).

Apesar da perda de dados durante a conversão de MARC 21 para MODS, Taylor e Joudrey (2009, p. 153) destacam que a alta compatibilidade entre o MARC 21 e o MODS e a provisão de dados mais ricos e estruturados deste esquema de metadados, pode, eventualmente, fazer dele o escolhido pelas bibliotecas como um meio para a descrição de recursos informacionais em ambientes $\mathrm{XML}$ em detrimento do padrão Dublin Core.

Outro fato destacado por Taylor e Joudrey (2009, p. 153) é que o MODS utiliza tags (etiquetas) baseadas em palavras, o que o torna mais amigável aos seus usuários. Nos formatos MARC 21 as tags dos campos são compostas por três dígitos numéricos, enquanto que as tags dos subcampos são compostas por apenas um caractere alfanumérico.

Para complementar o MARC 21 bibliográfico, surgiu o MARC 21 de autoridade. O MARC 21 de autoridade acompanhou O MARC 21 bibliográfico durante os esforços realizados pela LC para permitir a utilização dos registros MARC 21 em ambientes XML.

Tendo como base a relação entre registro bibliográfico e registro de autoridade e a relação entre o MARC 21 bibliográfico e o MARC 21 de autoridade, foi desenvolvido o Metadata Authority Description Schema (MADS) (Esquema de Metadados para a Descrição de Autoridade) como um apoio à utilização do MODS.

O Metadata Authority Description Schema (MADS) é um esquema com um conjunto de elementos de autoridade que pode ser utilizado para prover metadados sobre agentes (pessoas, organizações), eventos e termos (tópicos, geográficos, gêneros, etc.). O MADS serve como um acompanhamento do Metadata Object Description Schema (MODS) para prover metadados sobre as entidades autorizadas utilizadas nas descrições em MODS (LIBRARY OF CONGRESS, 2011d, tradução nossa). 
Cabe ressaltar que, embora o MADS tenha surgido para acompanhar 0 MODS, ambos os padrões podem ser utilizados de forma independente.

A versão preliminar do MADS foi lançada em abril de 2004, em dezembro do mesmo ano foi divulgado um novo rascunho e em abril de 2005 foi publicada a versão 1.0. Em junho de 2011 foi publicada a versão 2.0 do padrão. Atualmente o MADS está sob a responsabilidade do MODS/MADS Editorial Committee por meio do Network Development and MARC Standards Office da LC (MCCALLUM, 2005; LIBRARY OF CONGRESS, 2010a, 2011a).

O MADS e o MODS compartilham algumas características:

- Podem ser utilizados para comportar dados de registros MARC 21 ou para criação de registros originais;

- Incluem elementos selecionados dos formatos MARC 21;

- Seus registros podem ser convertidos por meio de folhas de estilo;

- Utilizam tags baseadas em palavras.

Para uma melhor compreensão do MADS, é apresentado na Figura 10 exemplo de um registro MADS. 
Figura 1 - Exemplo de registro MADS

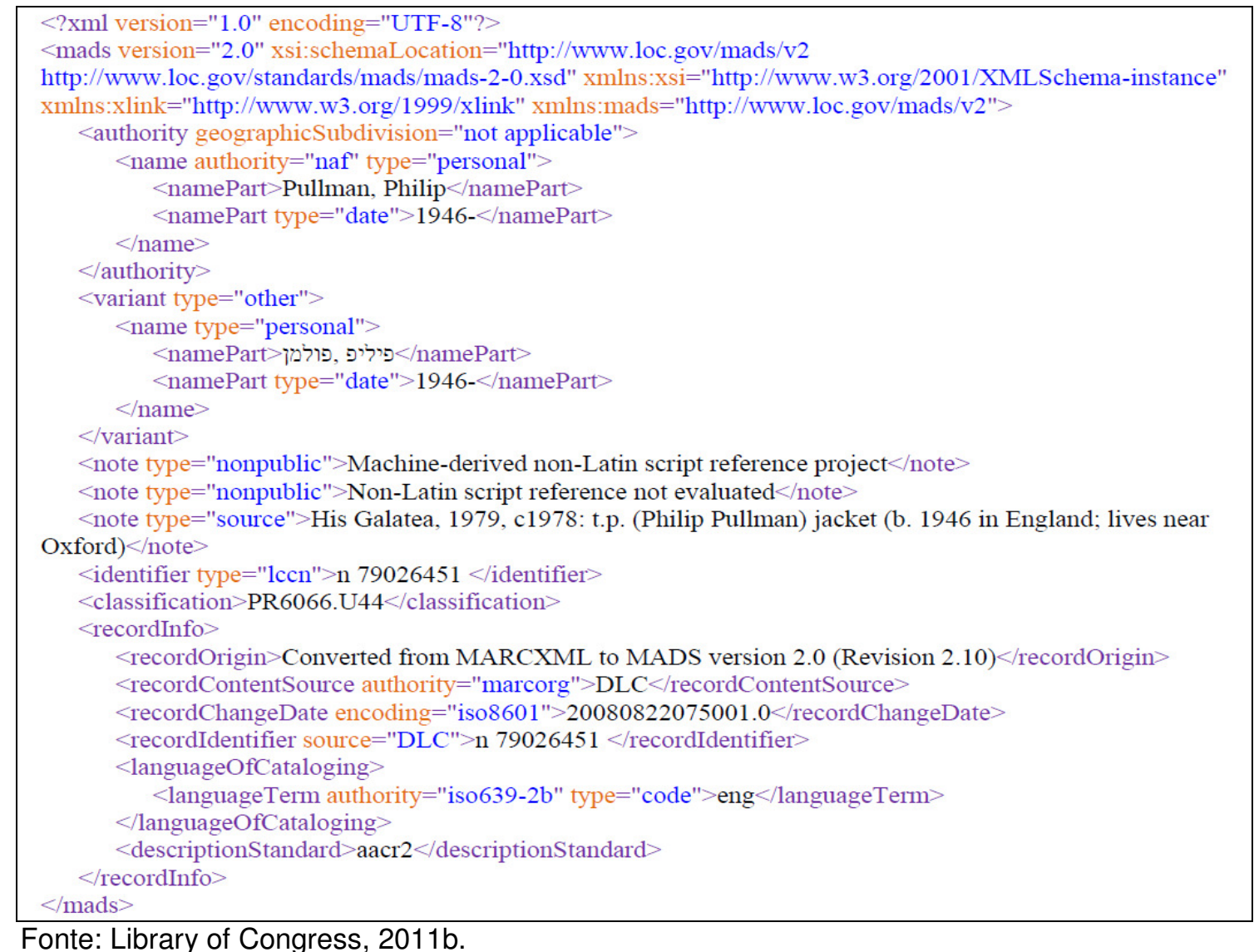

Em âmbito internacional algumas instituições já utilizam o MADS como uma alternativa para a exportação de seus registros de autoridade, são exemplos dessas instituições a LC (http://id.loc.gov), a Library and Archives Canada (http://www.collectionscanada.gc.ca/databases/canadiana-authorities) e a Biblioteca Virtual de Andalucía (http://www.bibliotecavirtualdeandalucia.es). No decorrer do desenvolvimento deste estudo não foram encontradas instituições nacionais que utilizam o MADS.

\section{ESTRUTURA DE UM REGISTRO MADS}

Um documento MADS, ou seja, um documento XML que está estruturado de acordo com o MADS, pode conter um único registro de autoridade ou uma coleção de registro de autoridade. No primeiro caso, o elemento raiz - elemento que compreende todo o conteúdo de um documento XML - será o elemento mads, enquanto que no segundo será madsCollection, como mostrado na Figura 2. 
Fabrício Silva Assumpção; Plácida Leopoldina Ventura Amorim da Costa Santos

Metadata authority description schema (MADS): uma alternativa à utilização do formato MARC 21 para dados de autoridade

Figura 2 - Exemplos da utilização dos elementos mads e madsCollection.

Documento com um registro MADS

$<$ ?xml version="1.0" encoding="UTF-8"?>

$<$ mads $>$

Registro de autoridade

$</$ mads $>$
Documento com mais de um registro MADS

$<$ ?xml version="1.0" encoding="UTF-8"?>

$<$ madsCollection $>$

$<$ mads>

Registro de autoridade

$</$ mads $>$

$<$ mads $>$

Registro de autoridade

$</$ mads $>$

$<$ mads $>$

Registro de autoridade $<$ /mads $>$

$<$ /madsCollection $>$

Fonte: Elaborado pelos autores

O elemento mads pode conter dois tipos de elementos: os elementos principais e os elementos secundários.

\subsection{Elementos Principais}

Os elementos principais que podem existir em um registro MADS são: authority, related e variant. Em um registro, é obrigatória a presença de, no mínimo, um elemento authority, já os elementos related e variant são opcionais.

O elemento authority inclui os elementos que formam o ponto de acesso autorizado para uma pessoa, organização, evento, título ou termo (tópico, gênero, geográfico). Em um registro MADS, o elemento authority pode ser repetido somente se prover pontos de acesso autorizados em diferentes idiomas ou alfabetos.

Junto ao elemento authority pode ser utilizado 0 atributo geographicSubdivision, o qual indica se o ponto de acesso autorizado pode ou não receber uma subdivisão geográfica, por exemplo, o nome de um país, jurisdição, região ou característica geográfica.

O elemento related inclui os elementos que formam o ponto de acesso autorizado da entidade que está associada à entidade descrita no registro MADS. Esse elemento equivale às remissivas "ver também" dos catálogos, podendo ser 
repetido em um registro quantas vezes forem necessárias. Um de seus atributos é type, que indica o tipo de relacionamento existente: earlier, later, parentOrg, broader, narrower, equivalent ou other.

O elemento variant inclui um ponto de acesso não autorizado (remissiva "ver" ou forma variante do nome) para a entidade descrita no registro, assim, pode ser repetido em um registro quantas vezes forem necessárias. Um dos atributos do elemento variant é type. Os valores definidos para o atributo type são acronym, abbreviation, translation, expansion e other.

Alguns elementos do MADS podem ocorrer como subelementos (elementos hierarquicamente subordinados) de qualquer um dos elementos principais. Esses elementos, chamados de elementos descritores, são:

- name: utilizado para nomes de pessoas, entidades coletivas, eventos, conferências ou famílias. O atributo type especifica o tipo de nome: personal, corporate e conference. O elemento name possui dois subelementos: namePart e description. Esses subelementos podem ocorrer mais de uma vez caso seja necessário. O tipo do atributo namePart pode ser especificado por meio dos valores do atributo type: date, family, given e termsOfAddress.

- titlelnfo: utilizado para títulos uniformes (pontos de acesso autorizados representando obras e expressões). Os subelementos desse elemento são title, subTitle, partNumber, partName e nonSort. Tais subelementos são utilizados para cada um dos elementos que podem compor um título uniforme.

- topic: utilizado para pontos de acesso de assunto.

- temporal: utilizado para pontos de acesso de assunto.

- genre: utilizado para pontos de acesso que representam gêneros.

- geographic: utilizado para pontos de acesso de nomes geográficos.

- hierarchicalGeographic: utilizado para prover um ponto de acesso autorizado para nomes geográficos hierárquicos. Como subelementos desse elemento podem ser utilizados os elementos continent, country, province, region, state, territory, county, city, island, area, extraterrestrialArea e citySection.

- occupation: utilizado para pontos de acesso que representam profissões. 
Em um registro em MADS, o elemento authority deve ter como subelemento, no mínimo, um elemento descritor. Os casos em que o elemento authority tem mais de um elemento descritor incluem, dentre outros, os registros que possuem pontos de acesso autor/título e, assim, necessitam dos elementos descritores name e titlelnfo.

Além dos elementos principais, nos quais são utilizados os elementos descritores, podem ocorrer em um registro MADS os chamados elementos secundários.

\subsection{Elementos Secundários}

Em um registro MADS, além dos elementos essenciais (authority, related e variant), podem ser acrescentados os elementos secundários: affiliation, classification, fieldOfActivity, identifier, language, note, url, extension e recordlnfo. Tais elementos complementam o registro e não possuem um número máximo de ocorrências.

- affiliation: utilizado para informações de contato ou sobre a afiliação de uma pessoa ou entidade coletiva.

- classification: inclui uma designação que é aplicada a um recurso indicando seu assunto, tal como uma notação de assunto construída com a Classificação Decimal de Dewey (CDD) ou com a Classificação Decimal Universal (CDU).

- fieldOfActivity: inclui um campo de atuação ou de negócios ou uma área de competência de uma pessoa ou entidade coletiva.

- identifier: inclui um identificador associado à entidade descrita no registro. O atributo type especifica o esquema utilizado para atribuir o identificador. Se o identificador for, por exemplo, o número de controle da LC, o valor do atributo type deve ser "Iccn".

- language: utilizado para indicar o idioma do registro de autoridade. Os subelementos do elemento language são languageTerm e scriptTerm, que especificam, respectivamente, a idioma e o alfabeto utilizado no registro de autoridade.

- note: utilizado para conter informações gerais. 
- url: inclui qualquer URL associado à entidade descrita no registro (por exemplo, o web site de uma entidade coletiva ou de uma pessoa).

- extension: utilizado para registrar dados não cobertos pelo MADS, por exemplo, dados que são úteis somente no contexto de uma determinada agência catalogadora.

- recordlnfo: inclui subelementos relacionados ao registro, e não à entidade descrita: descriptionStandard, recordContentSource, recordCreationDate, recordChangeDate, recordldentifier, recordOrigin e languageOfCataloging.

Como mencionado anteriormente, o MADS pode ser utilizado também para comportar dados de registros de autoridade MARC 21. Para tanto é necessário converter tais registros MARC 21 em registros MADS.

\section{CONVERSÃO DE REGISTROS MARC 21 PARA REGISTROS MADS}

A conversão de registros de autoridade no formato MARC 21 para registros MADS tem como intermediário o MARCXML. A conversão de registros MARC 21, estruturados de acordo com a ISO 2709, para registros MARC 21 em MARCXML e vice-versa pode se dar por meio do MARCXML Toolkit, disponibilizado pela LC (LIBRARY OF CONGRESS , 2011b), ou por meio de utilitários como o MarcEdit (http://people.oregonstate.edu/ reeset/marcedit/html/index.php). Uma vez que o MARCXML permite a codificação completa de registros MARC 21 em XML, essa conversão não causa perdas nos dados registrados nos campos, subcampos e indicadores do registro MARC 21.

Uma vez que o registro de autoridade está de acordo com o MARCXML, é possível convertê-lo em um registro MADS utilizando a folha de estilo "MARCXML Authorities to MADS Stylesheet" (http://www.loc.gov/standards/marcxml/xslt/MARC21slim2MADS.xsl) em qualquer utilitário que processe documentos XML e transforme-os utilizando folhas de estilo. O processo de conversão MARC 21 (ISO 2709) para MADS e vice-versa pode ser demonstrado pelo diagrama da Figura 3. 
Figura 3 - Conversão de registros MARC 21 para registros MADS.

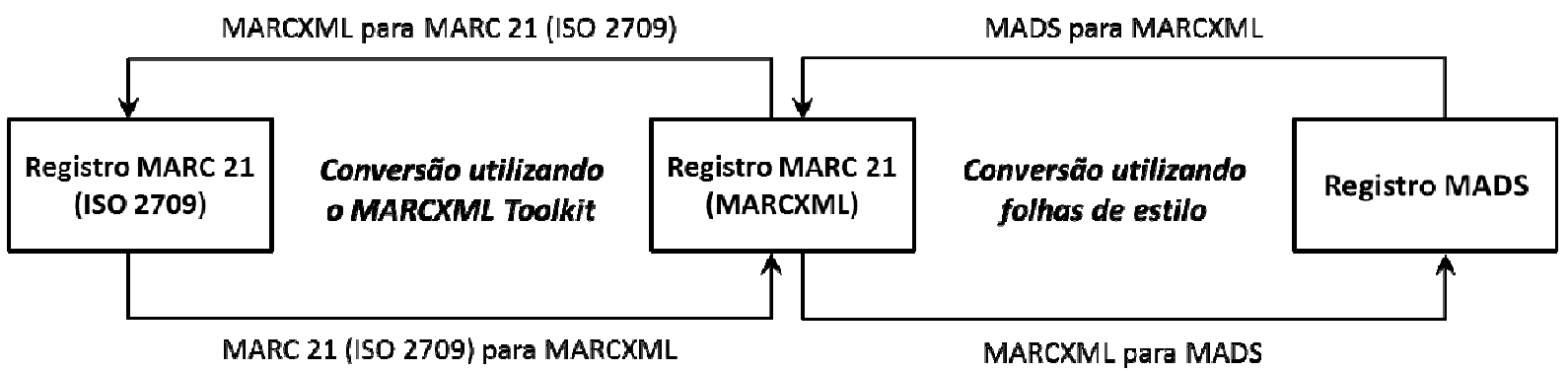

Fonte: Elaborado pelos autores

Como mencionado anteriormente, o MADS inclui um subconjunto dos elementos permitidos no Formato MARC 21 para Dados de Autoridade, ou seja, nem todos os campos, subcampos e indicadores do MARC 21 de autoridade possuem equivalentes no MADS, fato que pode ocasionar a perda ou a junção de dados após a conversão de um padrão para outro.

Um dos elementos permitidos pelo MARC 21 de autoridade é a forma completa do nome de uma pessoa, que é registrada no subcampo "q" do campo 100. No MADS, o elemento utilizado para registrar nomes de pessoas é o elemento name, que tem como subelementos namePart e description. $\mathrm{O}$ tipo do elemento namePart pode ser especificado por meio do atributo type e de seus valores (date, family, given, termsOfAddress). Assim, não há no MADS um elemento ou atributo para indicar a forma completa do nome de uma pessoa.

Durante a conversão de um registro MARC 21 que possui o subcampo "q" do campo 100 para um registro MADS, existem três opções em relação ao que pode ser feito com os dados registrados no subcampo "q":

1) unir os dados do subcampo "q" aos dados do subcampo "a" (nome pessoal);

2) manter os dados do subcampo "q" isolados dos dados do subcampo "a" em um elemento namePart; ou

3) descartar os dados do subcampo "q".

Essas três opções são exemplificadas na Figura 4. 
Figura 4 - Opções na conversão do subcampo "q" do campo 100.

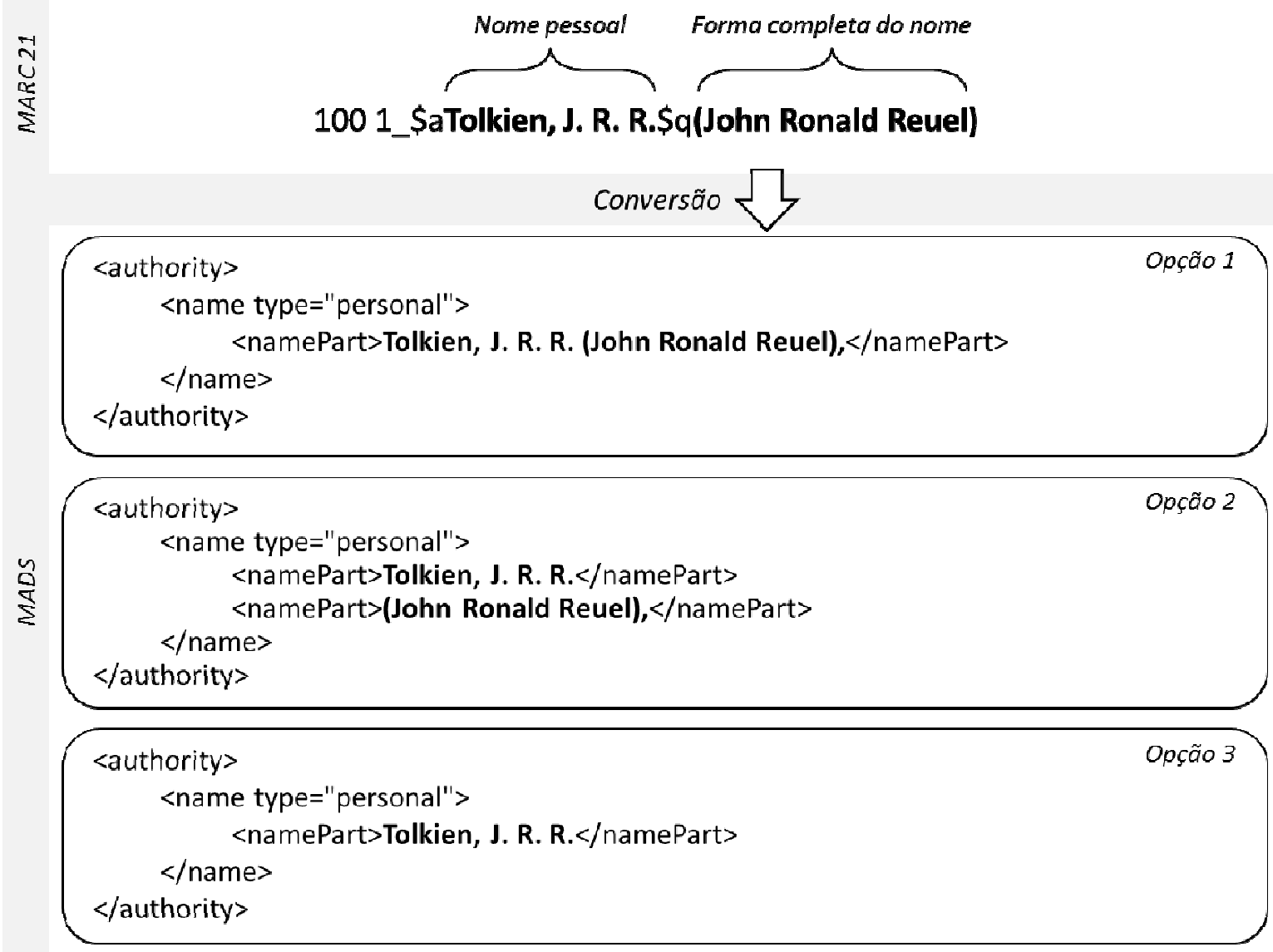

Fonte: Elaborado pelos autores

Visto que a perda ou a alteração de dados pode ocorrer na conversão de um padrão para outro, o que as instituições que optarem pela utilização do MADS devem buscar são meios (por exemplo, folhas de estilo) que permitam a conversão com o mínimo possível de danos ao registro de autoridade, permitindo seu intercâmbio e sua utilização entre instituições que adotam padrões de estrutura de dados diferentes.

\section{CONSIDERAÇÕES FINAIS}

Os registros de autoridade são os componentes essenciais para que o controle de autoridade (consistência dos pontos de acesso) seja alcançado em um catálogo. Uma vez tais registros são criados, podem ser intercambiados entre as agências catalogadoras, reduzindo os custos com a catalogação. Para possibilitar 
esse intercâmbio, uma das exigências é a criação e a adoção de padrões de estrutura de dados.

Para o intercâmbio de registros de autoridade são destacados dois padrões: 0 MARC 21 Format for Authority Data (Formato MARC 21 para Dados de Autoridade), que descende do Formato MARC criado na década de 1960, e o Metadata Authority Description Schema (MADS), criado com base na Extensible Markup Language (XML).

Algumas das características do MADS - ter com base a XML, permitir a criação de registros de autoridade simples e comportar alguns dos elementos do Formato MARC 21 de autoridade - o tornam um padrão mais apto, se comparado ao MARC 21, para ser utilizado no ambiente Web e além do contexto das bibliotecas.

Como demonstrado no item 5, o MADS possui algumas limitações no que diz respeito a sua capacidade de comportar dados de registros MARC 21. No entanto, são essas limitações, principalmente decorrentes da falta de especificidade no registro de alguns dados, que caracterizam o MADS como um padrão a ser utilizado para a criação de registros de autoridade mais simples que os exigidos pelos altos padrões das agências bibliográficas nacionais, como a Library of Congress, por exemplo.

O nível de especificidade provido pelo MADS mostra-se suficiente para a criação de registros de autoridade com o nível de funcionalidade requerido por grande parte das agências catalogadoras, por exemplo, as bibliotecas universitárias. Esse nível de funcionalidade muitas vezes inclui somente o ponto de acesso autorizado, as remissivas "ver" e "ver também" e alguma nota de escopo ou fontes de informação consultadas para a criação do registro.

A literatura sobre o MADS, assim como sobre o MODS, ainda é escassa, o que vêm a desfavorecer o seu conhecimento e a sua utilização por parte das agências catalogadoras. No contexto dos avanços proporcionados pelas Tecnologias de Informação e Comunicação, os estudos sobre padrões de metadados são essenciais, uma vez que tais padrões estão relacionados à interoperabilidade, conceito chave para os atuais sistemas de informação.

Além da escassez de publicações sobre o MADS, é perceptível também a falta de estudos nacionais sobre o controle de autoridade na catalogação descritiva, sobre as atividades que visam a alcançar tal controle e sobre suas implicações. A 
falta de tais estudos aponta uma lacuna na literatura nacional em uma temática que, frente à proposta da Web Semântica e ao papel da Ciência da Informação na construção e análise dos ambientes informacionais digitais mostra-se de caráter emergencial.

\section{REFERÊNCIAS}

ALVES, Rachel Cristina Vesú. Metadados como elementos do processo de catalogação. 2010. 132 f. Tese (Doutorado em Ciência da Informação) - Faculdade de Filosofia e Ciências, Universidade Estadual Paulista, Marília, 2010. Disponível em:

$<$ http://www.athena.biblioteca.unesp.br/exlibris/bd/bma/33004110043P4/2010/alves_ rcv_dr_mar.pdf>. Acesso em: 16 jun. 2012.

BURGER, Robert. Authority work: the creation, use, maintenance, and evaluation of authority records and files. Littleton: Libraries Unlimited, 1985.

FLAMINO, Adriana Nascimento. MARCXML: um padrão de descrição para recursos informacionais em Open Archives. 2006. 164 f. Dissertação (Mestrado em Ciência da Informação) - Faculdade de Filosofia e Ciências, Universidade Estadual Paulista, Marília, 2006. Disponível em:

<http://www.athena.biblioteca.unesp.br/exlibris/bd/bma/33004110043P4/2006/flamin o_an_me_mar_prot.pdf >. Acesso em: 16 jun. 2012.

LIBRARY OF CONGRESS. MADS schema. Version 1.0. Washington, 2010a.

Disponível em: <http://www.loc.gov/standards/mads/mads-1-0.xsd>. Acesso em: 1 dez. 2011.

. MADS schema. Version 2.0. Washington, 2011a. Disponível em: <http://www.loc.gov/standards/mads/mads.xsd>. Acesso em: 1 dez. 2011.

MADS. <http://lccn.loc.gov/n79026451/mads>. Acesso em: 4 dez. 2011b.

.MARC 21 XML schema: official web site. Washington, 2011c. Disponível em: <http://www.loc.gov/standards/marcxml/>. Acesso em: 1 dez. 2011.

Metadata authority description schema: official web site. Washington, 2011d. Disponível em: <http://www.loc.gov/standards/mads/>. Acesso em: 1 dez. 2011.

MODS: uses and features. Washington, 2010b. Disponível em: <http://www.loc.gov/standards/mods/mods-overview.html . Acesso em: 1 dez. 2011. 
MARAIS, Hester. Authority control in an academic library consortium using a union catalogue maintained by a central office for authority control. 2004. $310 \mathrm{f}$. Tese (Doctor of literature and philosophy in the subject Information Science) University of South Africa, Petroria. 2004. Disponível em: <http://uir.unisa.ac.za/bitstream/10500/2546/1/thesis.pdf>. Acesso em: 16 jun. 2012.

MCCALLUM, S. MADS, a MODS Companion. 2005. Disponível em: $<$ http://www.ala.org/ala/mgrps/divs/lita/litamembership/litaigs/authorityalcts/MADS200 5Annual.ppt>. Acesso em: 24 abr. 2011.

MILLER, Dick R.; CLARKE, Kevin S. Putting XML to work in the library: tools for improving access and management. Chicago: American Library Association, 2004.

MORENO, Fernanda Passini; BRASCHER, Marisa. MARC, MARCXML e FRBR: relações encontradas na literatura. Informação \& Sociedade: Estudos, João Pessoa, v. 17, n. 3, p. 13-25, set./dez. 2007. Disponível em: <http://www.ies.ufpb.br/ojs2/index.php/ies/article/view/834>. Acesso em: 16 jun. 2012.

RAY, Erik T. Learning XML. 3rd ed. Beijing: O'Reilly, 2003.

REITZ, Joan M. Online dictionary for library and information science. Last updated March 9, 2010. Santa Barbara: Libraries Unlimited, 2010. Disponível em: <http://lu.com/odlis/>. Acesso em: 10 nov. 2010.

SIQUEIRA, Marcos Antonio. XML na Ciência da Informação: uma análise do MARC 21. 2003. 133 f. Dissertação (Mestrado em Ciência da Informação) Faculdade de Filosofia e Ciências, Universidade Estadual Paulista, Marília, 2003. Disponível em: <http://www.athena.biblioteca.unesp.br/exlibris/bd/bma/33004110043P4/2003/siqueir a_ma_me_mar.pdf>. Acesso em: 16 jun. 2012.

INTERNATIONAL FEDERATION OF LIBRARY ASSOCIATIONS - IFLA. Statement of international cataloguing principles. 2009. Disponível em: <http://www.ifla.org/files/cataloguing/icp/icp_2009-en.pdf>. Acesso em: 16 jun. 2012.

TAYLOR, Arlene G.; JOUDERY, Daniel N. The organization of information. $3^{\text {th }}$ ed. Westport: Libraries Unlimited, 2009.

W3C. Extensible markup language (XML). 2011. Disponível em: <http://www.w3.org/XML>. Acesso em: 14 ago. 2011.

\section{Title}

Metadata Authority Description Schema (MADS): an alternative to MARC 21 Format for Authority Data

Abstract 
Introduction: Authority records interchange requires establishing and using metadata standards, such as MARC 21 Format for Authority Data, format used by several cataloging agencies, and Metadata Authority Description Schema (MADS), that has received little attention and it is a little widespread standard among agencies.

Objetive: To present an introductory study about MADS standard.

Methodology: Descriptive and exploratory bibliographic research.

Results: The paper address the MADS creation context, its goals, structure as well as critical issues related to conversion of records from MARC 21 to MADS.

Conclusions: Concludes that MADS, despite its limitations, may be used to create simple authority records in Web environment and beyond libraries context.

Key-words: Metadata Authority Description Schema (MADS). Extensible Markup Language $(\mathrm{XML})$. MARC 21 Format for authority data. Authority control. Descriptive cataloging.

\section{Título}

Metadata Authority Description Schema (MADS): una alternativa al uso del Formato MARC 21 para Datos de Autoridad

\section{Resumen}

Introducción: El intercambio de registros de autoridad requiere el establecimiento y adopción de estándares de metadatos como el Formato MARC 21 para Datos de Autoridad, formato utilizado por varias agencias de catalogación, y el Metadata Authority Description Schema (MADS), un estándar que sigue siendo en gran parte inexplorado por la literatura y poco conocido entre las agencias.

Objetivo: Presentar un estudio introductorio sobre el estándar MADS.

Metodología: Investigación bibliográfica descriptiva y exploratoria.

Resultados: El estudio aborda el contexto de la creación del MADS, sus objetivos, su estructura y los temas clave relacionados con la conversión de los registros MARC 21 para registros MADS.

Conclusiones: El estudio concluye que el MADS, a pesar de sus limitaciones, puede ser utilizado para la creación de registros de autoridad sencillos, en el entorno Web y más allá del contexto de las bibliotecas.

Palabras Clave: Metadata Authority Description Schema (MADS). Extensible Markup Language (XML). Formato MARC 21 para Datos de Autoridad. Control de autoridad. Catalogación descriptiva. Información y tecnología.

Recebido em: 19.06 .2012

Aceito em: 10.05.2013 